\title{
Exploring the immune-checkpoint inhibitors' efficacy/tolerability in special non-small cell lung cancer (NSCLC) populations: focus on steroids and autoimmune disease
}

\author{
Francesco Passiglia, Valeria Cetoretta, Marco De Filippis, Valerio Napoli, Silvia Novello \\ Department of Oncology, S. Luigi Gonzaga Hospital, University of Turin, Orbassano (TO), Italy \\ Contributions: (I) Conception and design: F Passiglia, S Novello; (II) Administrative support: V Cetoretta, M De Filippis; (III) Provision of study \\ materials or patients: F Passiglia, V Cetoretta, M De Filippis, V Napoli; (IV) Collection and assembly of data: V Cetoretta, M De Filippis; (V) \\ Data analysis and interpretation: F Passiglia, V Cetoretta, M De Filippis, S Novello; (VI) Manuscript writing: All authors; (VII) Final approval of \\ manuscript: All authors. \\ Correspondence to: Francesco Passiglia, MD. Department of Oncology, S. Luigi Gonzaga Hospital, University of Turin, Regione Gonzole, 10 , 10034 \\ Orbassano (TO), Italy. Email: francesco.passiglia@unito.it.
}

\begin{abstract}
The advent of immune-checkpoint inhibitors targeting the programmed cell death-1 (PD-1)/ programmed death ligand-1 (PD-L1) axis, both as monotherapy and in combination strategies, produced a paradigm change of the treatment algorithm for metastatic, non-oncogene addicted, non-small cell lung cancer (NSCLC) patients. Although the great efficacy and the optimal tolerability emerging from clinical studies has been confirmed for the majority of patients treated in the real-word scenario, however the potential activity and safety profile of these agents in uncommon NSCLC populations remains still controversial. Particularly, patients with previously diagnosed autoimmune disease or concomitant steroids treatment at the time of immunotherapy initiation represent two special subgroups of patients not unusual in the real-word practice, to whom the clinical implication of immune-checkpoint inhibitors administration is largely unknown. In this review we provided an updated literature overview, summarizing available evidence and reporting practical suggestions, which may guide physicians in their clinical management of these NSCLC sub-populations.
\end{abstract}

Keywords: Immunotherapy; programmed cell death-1/programmed death ligand-1 (PD-1/PD-L1); non-small cell lung cancer (NSCLC); steroids; autoimmune disease (AID)

Submitted May 06, 2020. Accepted for publication Oct 09, 2020.

doi: $10.21037 /$ tlcr-20-635

View this article at: http://dx.doi.org/10.21037/tlcr-20-635

\section{Introduction}

The advent of immunotherapy dramatically changed the therapeutic landscape of non-oncogene addicted metastatic non-small cell lung cancer (NSCLC). In the last few years, check-point inhibitors (CPIs) targeting the programmed cell death-1 (PD-1)/programmed death ligand-1 (PD-L1) axis, have shown a significant superiority over chemotherapy, in terms of efficacy and tolerability, improving disease control, overall survival (OS) and quality of life (QoL), both in first- and second-line setting (1-7). Based on the Check-Mate 017/057 and OAK clinical trials' results, nivolumab and atezolizumab entered in the clinical practice for the second/third-line treatment of PD-L1 unselected NSCLC patients, while the KEYNOTE-010 and 024 studies granted Pembrolizumab approval as new standard of care for both pre-treated and untreated NSCLC patients with tumor PD-L1 expression of at least $1 \%$ and $50 \%$, respectively. The introduction of immunotherapy into clinical practice produced a significant increase of longterm survival, reaching $16 \%$ at 5 years in pre-treated, PDL1 unselected population, and about $30 \%$ when considering naïve patients with high PD-L1 expression, as compared 
to $5.5 \%$ in the chemotherapy era (8). More recently, the combination of Pembrolizumab with first-line platinumbased chemotherapy resulted in a significant increase of tumor responses and survival outcomes compared to chemotherapy alone (9-11), regardless of tumor histology and PD-L1 expression levels, thus emerging as an additional effective option for the treatment of non-oncogene addicted, metastatic NSCLC. Together with the great efficacy, CPIs are characterized by an optimal tolerability profile, thus allowing their administration for prolonged periods. However, these drugs are not free of side effects, mostly due to the abnormal activation of the immune system. In fact, it is estimated that about $70 \%$ of patients develop adverse events during the course of CPIs therapy, usually not disabling and well manageable with medical therapy or drug withdrawal (12). Considering that the majority of available evidence is limited to highly selected population which reflects the ones included into clinical trials, few data are available from the real-life setting. Some clinical series have recently confirmed an optimal efficacy and tolerability of CPIs in special subgroups of patients, like elderly (13), poor performance status (14), and brain metastasis (15), who are quite common in the real-word scenario. Conversely, the potential activity and safety profile of CPIs in NSCLC patients who received a concomitant use of steroids or were previously diagnosed with autoimmune disease (AID) remains still controversial. In this review we provided an updated literature overview, summarizing available evidence and reporting practical suggestions, which may guide physicians in their current practice.

\section{Concomitant use of steroids}

\section{Clinical evidences}

Glucocorticoids are widely used for the clinical management of advanced NSCLC patients to treat both cancer-related and cancer unrelated symptoms (16-20). The most common cancer-related palliative indications include dyspnea, fatigue, anorexia, pain and symptomatic brain metastases. Otherwise patients could require steroids therapy administration for the clinical management of a wide range of conditions, like AID, chronic obstructive pulmonary disease (COPD) flare, prophylaxis of hypersensitivity reactions, and non-cancer pain.

Steroids exert their dose-dependent immunosuppressive effects both on innate and adaptive immunity. Wellestablished effects include the induction of $\mathrm{T}$ cell apoptosis and the maturation impairment of dendritic cells (DCs) (21). Moreover, some pre-clinical works demonstrated that low dose glucocorticoids administration is sufficient to suppress response to cancer immunotherapy (22). The raising concern on the immunosuppressive effect of glucocorticoids have led to a formal exclusion of patients receiving $>10 \mathrm{mg} /$ day of prednisone or its equivalent, from the majority of CPIsbased randomized clinical trials (23).

Different publications have clearly suggested that a short course of glucocorticoids therapy does not significantly affect T-cells activity $(24,25)$. As a matter of fact, some retrospective studies showed that a transient use of steroids for the clinical management of immune-related adverse events (irAEs) do not compromise the efficacy of immunotherapy (26-31). Similarly, the use of steroids-based premedication in the record trials of recently approved chemo-immunotherapy combination regimens did not have any influence on patients' survival outcomes (9-11).

Conversely, the potential effect of a concomitant administration of steroids at the time of CPIs initiation in cancer patients remains still controversial. Several retrospective studies and case series were carried out with the intent to clarify the potential clinical implications of concomitant use of steroids in CPIs-treated, advanced NSCLC patients (Table 1).

Arbour et al. analyzed 640 patients who were treated with anti-PD-1/PD-L1 agents coming from the Memorial Sloan Kettering Cancer Centre (MSKCC; 455) and the Gustave Roussy Cancer Centre (GRCC; 185). In this study, 90 patients $(14 \%)$ were treated with a baseline $>10 \mathrm{mg}$-dose of prednisone at the time of CPIs beginning, and treatment outcomes, including objective response rate (ORR), progression free survival (PFS), and OS, were analyzed. Main factors considered in the multivariate analysis included smoking status, Easter Cooperative Oncology Group performance status (ECOG-PS) and the presence of brain metastases. The pooled analysis showed that $\geq 10 \mathrm{mg}-$ prednisone administration at early stages of CPIs' therapy was significantly associated to poor survival outcomes, specifically in terms of PFS (HR 1.31, $\mathrm{P}<0.03$ ), and OS (HR 1.66, $\mathrm{P}<0.001)$. A detrimental effect of steroids on CPIs' efficacy has been observed in patients receiving $\geq 10 \mathrm{vs}$. $<10 \mathrm{mg}$ prednisone (32).

In a second retrospective study, Scott $e t$ al. analyzed 210 NSCLC patients treated with nivolumab at the Cleveland Clinic, with $66(31 \%)$ receiving a concomitant steroids therapy. The median OS of patients subjected to steroids treatment within the first 30 days was 4.3 months, compared 


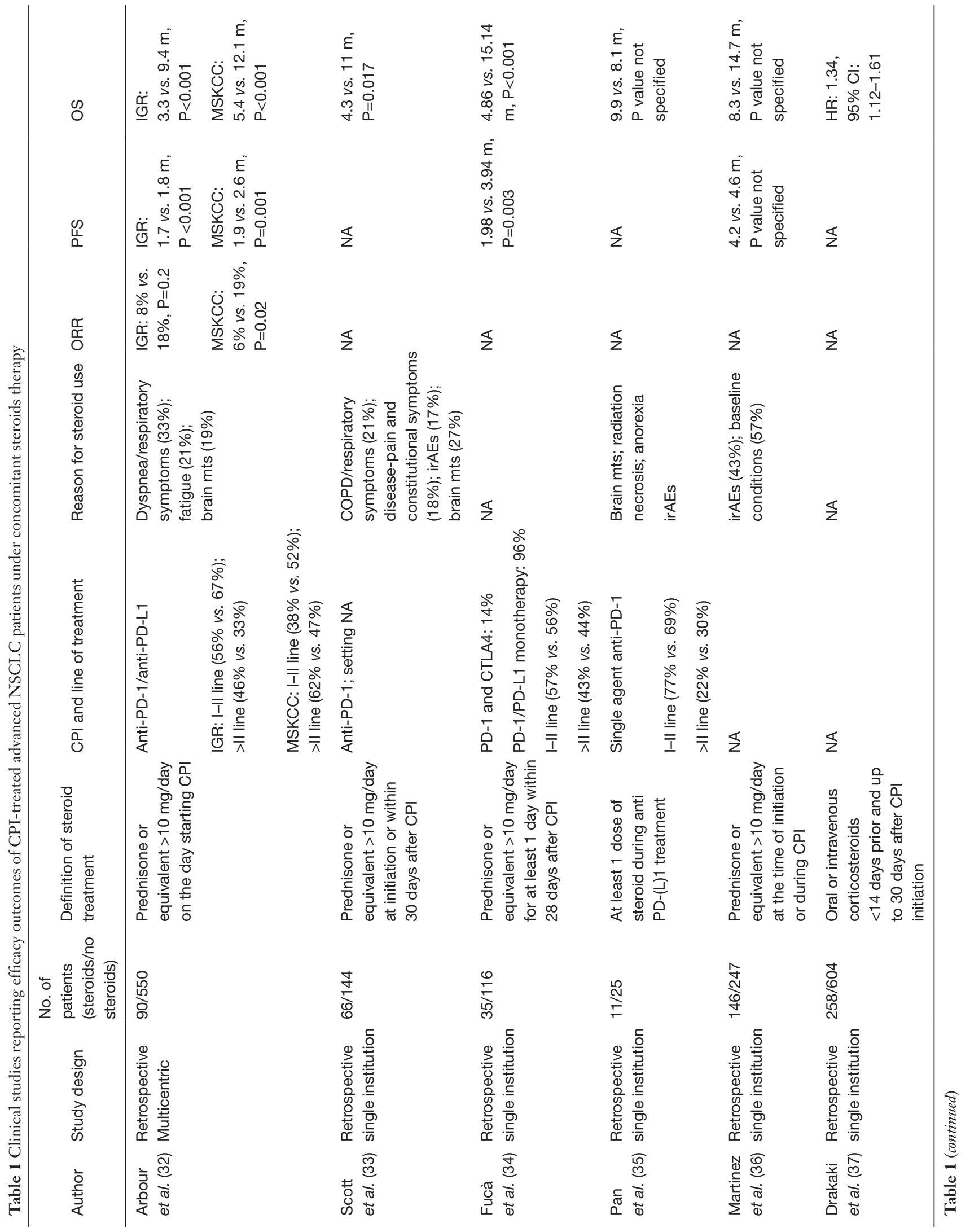




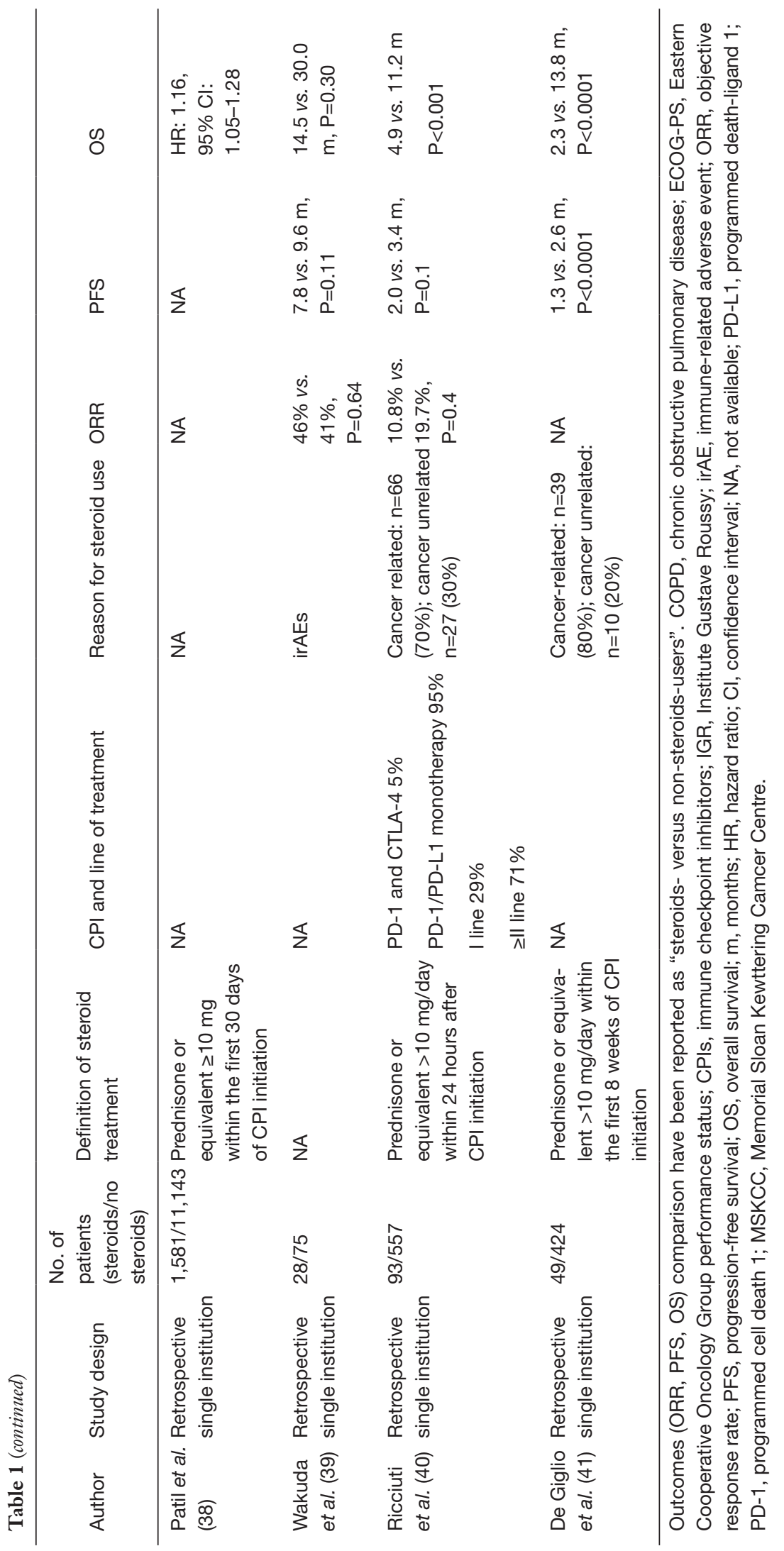


to 11-month OS of other patients (HR 2.30, 95\% CI: 1.27-4.16, $\mathrm{P}=0.006)$. Among them, 31 individuals receiving steroids for the clinical management of irAEs did not show significant differences in terms of OS as compared to those without irAEs (OS: 16.1 vs. 10.5 months; 95\% CI: 8.6-12.2, $\mathrm{P}=0.50)(33)$.

Fucà $e t$ al. conducted another retrospective analysis on a total of 151 patients diagnosed with metastatic NSCLC undergoing CPIs treatment. In this study, steroid exposure was defined as the $\geq 10 \mathrm{mg}$-prednisone administration in at least one of the 28 days preceding the CPIs' treatment initiation. The 35 steroid-exposed patients ( $23 \%$ of the overall population) showed significantly worse PFS (HR 1.80, $\mathrm{P}=0.003)$ and $\mathrm{OS}(\mathrm{HR} 2.60, \mathrm{P}<0.001)$ than the nonexposed cohort (34).

Numerous subsequent reports, which were published as abstracts in several congresses, appear to be aligned with the above-mentioned evidence, overall showing a detrimental effect of a concomitant use of steroids at baseline on CPIs' efficacy and patients' survival (Table 1) (35-38).

Of note the only report considering corticosteroids administration during CPIs' therapy exclusively devoted to the clinical management of irAEs showed no significant difference in terms of OS (14.5 vs. 30.0 months, $\mathrm{P}=0.30$; HR 0.69), PFS (7.8 vs. 9.6 months, $\mathrm{P}=0.11$; HR 0.65) and ORR ( $46 \%$ vs. $41 \%$; $\mathrm{P}=0.64$ ) between steroids and nonsteroids group, respectively (39).

Interestingly, two most recent studies, analyzed the effects of corticosteroids on CPIs' treatment efficacy considering the reason of their administration by splitting cancer-related palliative indications (brain metastases, dyspnea, bone metastases, anorexia) from cancer-unrelated indications (irAEs, COPD exacerbation, AID, pain, chemotherapy or iodinate contrast prophylaxis etc.).

In the retrospective study by Ricciuti et al., the poorest survival outcome likely applies to the subgroup of patients who received $10 \mathrm{mg}$-prednisone for palliative purposes. They identified a total of 650 CPI-treated, advanced NSCLC patients, of whom 93 received prednisone $\geq 10 \mathrm{mg}$ or its equivalent within 24 hours of CPI initiation. Among them, 66 patients were on steroids treatment for cancerrelated palliative symptoms, including symptomatic brain metastases $(57.6 \%)$, dyspnea (18.2\%), bone metastases $(16.7 \%)$, and anorexia (7.6\%), whilst 27 patients for cancer unrelated conditions. Patients who received $\geq 10 \mathrm{mg}$ of prednisone for palliative indications showed significantly worse survival outcomes compared to those receiving $\geq 10 \mathrm{mg}$ of prednisone for cancer unrelated indications or lower dose of steroids (PFS: 1.4 vs. 4.6 vs. 3.4 months, $\mathrm{P}=0.001$; OS: 2.2 vs. 10.7 vs. 11.2 months, $\mathrm{P}=0.001$ ). Conversely no survival differences were observed between patients receiving $\geq 10 \mathrm{mg}$ prednisone for non-cancer related indications and those on lower dose of steroids, both in terms of PFS (4.6 vs. 3.4 months; HR 0.77; 95\% CI: $0.50-1.19 ; \mathrm{P}=0.24)$ and $\mathrm{OS}(10.7$ vs. 11.2 months; HR 0.93; 95\% CI: 0.59-1.48; $\mathrm{P}=0.77)$ (40).

Similarly, De Giglio et al. performed a retrospective analysis on a total of 49 NSCLC patients who received early steroids therapy at Gustave Roussy Cancer Institute, whose 39 patients for cancer-related symptoms, including dyspnea $(50 \%)$, brain metastases $(15.8 \%)$, pain $(7.9 \%)$, superior vena cava syndrome $(7.9 \%)$, fatigue $(5.3 \%)$, others $(13.1 \%)$, and the remaining 10 patients for other causes, like the clinical management of irAEs (54.6\%). Patients receiving steroids for cancer-related symptoms had significantly poorer outcomes with early steroids introduction reported to be an independent prognostic factor for both poor PFS (HR 3.04; 95\% CI: 1.38-6.66; $\mathrm{P}=0.006)$ and OS (HR 1.21; 95\% CI: $0.53-2.8 ; \mathrm{P}<0.0001)$. No differences were observed between the group of patients subjected to steroids therapy for other indications [PFS 2.7 months (1.21-NR); OS 13.4 months (4.30-NR)] and the group of steroids-naïve patients [PFS 2.6 months (2.20-3.94); OS 13.8 months (11.4-18)] (41).

\section{Critical discussion}

Corticosteroids are immunomodulatory agents regulating gene expressions and signaling pathways through both genomic and non-genomic mechanisms $(21,42)$, thus exerting their dose-dependent immunosuppressive action on $\mathrm{T}$ cells, which constitute the effector arm of immunotherapy.

In the antitumor response, PD-1 exerts its inhibitory effects mainly at local level, in the tumor microenvironment (43) thus PD-1/PD-L1 CPIs aim to reverse the exhaustion of pre-existing tumor-residing T-cells, by restoring their effector functions, in order to produce tumor regression (44-46).

Nonetheless, it has not been clarified yet whether steroids administration block the differentiation of stimulated $\mathrm{T}$ cells or deplete already differentiated tumorreactive lymphocytes. Therefore, it remains unclear how steroids alter the adaptive anti-tumor immunity and whether steroids effects on immune response may vary according to their dosage, administration time-point/ duration, as well as on the basis of primary tumor location. 


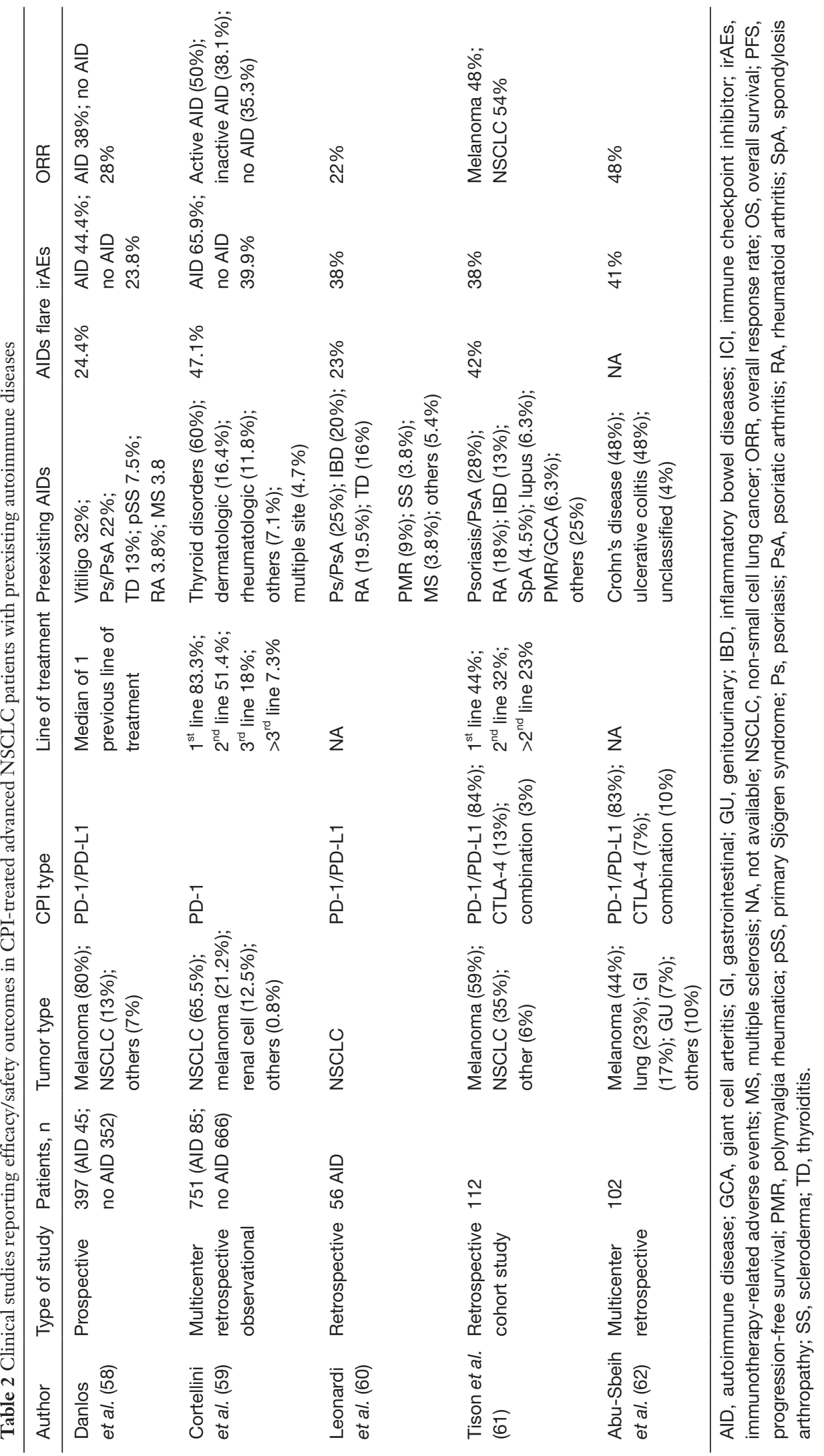


Although initial real-world clinical data (32) suggested a significant association between the dose of steroids administration and the therapeutic effects of CPIs, recent evidence $(34,40)$ revealed that the immunosuppressive effect of corticosteroids is more timely- than dose-dependent.

Several preclinical studies explored whether the dexamethasone administration timing may significantly affect immunotherapeutic treatment efficacy $(47,48)$. The results of these studies revealed that early steroid administration only, through the reduction of the $\mathrm{T}$ lymphocyte peripheral pool, might compromise the early adaptive immune response enhanced by anti-PD-1 therapy, thus impairing the clinical efficacy of these agents.

Most of the studies exploring the implications of steroids administration in NSCLC patients under CPIs' treatment, which are being considered in this instance, appear to support this item. Particularly, Fucà et al. provides a potential biological explanation to the early corticosteroid use negative effects on the antitumor immune response elicited by CPI therapy (34), showing as white blood count (WBC), absolute neutrophil count (ANC) and derived neutrophil to lymphocyte ratio (dNLR), were significantly higher in steroids-treated patients than the non-exposed cohort, both at baseline and at $4 / 6$ weeks after $\mathrm{CPI}$ initiation. Interestingly a high percentage of steroidstreated patients showed a NLR $\geq 5$ (OR 5.40, 95\% CI: $1.95-$ 16.70, $\mathrm{P}<0.001$ ) and a dNLR $\geq 3$ (OR 10.32, 95\% CI: $3.43-$ 39.43, $\mathrm{P}<0.001)$ as compared to the control cohort, thus suggesting as the dynamic modulation of peripheral blood immune cells during the treatment course might mediate immunotherapy resistance induced by corticosteroid administration.

Nonetheless, the relevance of the negative prognostic effect of steroids should be cautiously considered when they are used in NSCLC patients with metastatic disease. Indeed, when steroids administration was stratified and analyzed separately according to the specific clinical indication, the concomitant use of corticosteroids for non-palliative purposes did not appear to significantly affect CPIs-treated patients' survival anymore, regardless of their dose of administration $(40,41)$. Although limited by a retrospective design, low number and heterogeneity of included patients, the results of most recent studies suggested that the negative impact of steroids on CPIs' efficacy may be mainly ascribed to the worse prognosis of patients candidate to early palliative cure for their symptomatic disease. Moreover, subgroup analysis suggested that steroidtreated patients were commonly characterized by negative prognostic clinical features, such as ECOG-PS $\geq 2$, baseline brain metastases, and higher number of metastatic sites (32-34), thus confirming the predominant prognostic role of steroids at CPIs' therapy initiation.

On the other hand, once anti-tumor immunity has been initiated, the negative impact of corticosteroids on immune function is markedly reduced (47). In line with this hypothesis, neither steroid administration for irAEs treatment nor the short-course of steroid use within premedication protocol for chemo-immunotherapy combinations (9-11) significantly influenced the survival outcomes of NSCLC patients $(28,29,31)$.

Finally, preclinical studies suggested that blocking CTLA-4, but not PD-1, partially rescued T-cell proliferation in the presence of dexamethasone in vitro (49). This type of evidence is likely related to the different effect of CTLA-4 blockade, usually acting on less differentiated and more dexamethasone-sensitive T-cells subsets, suggesting a different effect of steroids on antiCTLA-4 and anti-PD-1/PD-L1 agents activity. Different studies have previously found that corticosteroids did not negatively impact OS of cancer patients on immunotherapy involving CTLA-4 blockade (28,50-54). Although none of these studies was specifically powered to address the impact of steroids on CPIs' efficacy, these evidences could partially reflect the anti-CTLA-4 specific capability of counteracting steroids effects. Conversely other clinical experiences suggested a potential detrimental effect of early steroids administration on the ipilimumab anti-tumor activity, requiring further investigation in dedicated studies (55).

Moving from bench to bedside, it will be interesting to see whether and how steroids use could affect the antiCTLA-4 plus anti-PD-1 blockade efficacy, since this kind of combination has been recently entered as additional first line treatment option for patients with metastatic, nononcogene addicted NSCLC, at least in the United States.

\section{Autoimmune diseases}

\section{Clinical evidences}

Among patients diagnosed with lung cancer, a percentage ranging from $14 \%$ to $25 \%$ is likely to have a concomitant AID (56). Female patients, elderly and early stage have higher chances of being part of this overlapping group $(\mathrm{P}<0.001)$. Moreover, it has been shown that some autoimmune disorders such as rheumatoid arthritis (RA), contribute to the onset of neoplasms. Indeed, subjects 
affected by RA present a double risk of developing lung cancer as compared to the healthy population, with cigarette smoking and lung chronic inflammation underlying this clinical association (57).

The concerns about exacerbations of pre-existing AID as well as the major susceptibility to severe irAEs as consequence of the reactivation of immune response induced by the checkpoint blockade led to the exclusion of patients with autoimmune disorders from the randomized phase III clinical trials leading to the approval of CPIs' therapy in lung cancer patients. However, different real word studies and retrospective series evaluated the potential clinical implications CPIs therapy in cancer patients with AIDs (Table 2).

A study published in 2018, including 172,285 patients with a lung cancer diagnosis performed between 1992 and 2009, showed that, in absence of CPIs' administration, the presence of a concomitant AIDs did not influence the treatment pattern and was not associated with an increased mortality (63).

In a prospective study based on REISAMIC (Registry of Severe Adverse events of Immunomodulating monoclonal antibodies in oncology), the association between preexisting AIDs and irAEs free survival, OS and best ORR was investigated, with 45 out of 397 enrolled cancer patients harboring a concomitant AIDs diagnosis. The most frequent AIDs were vitiligo $(n=17)$, psoriasis $(n=12)$, autoimmune thyroiditis $(n=7)$, Sjögren's syndrome $(n=4)$ and RA $(\mathrm{N}=2)$. The incidence of irAEs in patients with autoimmune pathologies was $44.4 \%$ compared to $23.8 \%$ of the remaining population. IrAEs median free survival was seen to be lower in patients with previous AIDs (5.4 months) compared to the control cohort (13 months). However, no OS and ORR differences were observed between the two groups (58). The study did not consider grade 1 immune-mediated toxicities in order to avoid detection bias, since the diagnose of mild immune-mediated toxicities is easier in patients with previous autoimmune disorders. On the other hand, this may have led to an underestimation of the incidence of irAEs. Furthermore, the authors didn't differentiate between clinically active and inactive AID at the time of anti PD-1 blockade initiation.

In another multicenter retrospective study published in 2019, among 751 stage IV cancer patients, the most represented tumor was NSCLC (65.5\%) followed by melanoma and kidney cancer. Seventy out of 751 had a pre-existing inactive AID, i.e., not being treated at the time of immunotherapy initiation, and 15 presented an active disease that required the administration of immunosuppressive drugs (corticosteroids in $73.3 \%$ of cases). The incidence of any grade irAEs in the population with previous AIDs was 65.9\% (95\% CI: 49.7-85.5\%), compared to $39.9 \%$ (95\% CI: $35.2-45.0 \%$ ) in patients without AIDs. However, not significant differences in the incidence of grade 3-4 irAEs [9.4\% (95\% CI: 4.1$18.5 \%$ ) vs. $8.8 \%$ (95\% CI: $6.7-11.4 \%$ )], ORR (38.1\% inactive $/ 50 \%$ active AID vs. $35.3 \%$ no AID), median PFS (14.4 inactive/6.8 months active AIDs vs. 8 months no AID) and median OS (15.7 inactive/9.8 months active AID vs. 16.5 months no AID) were reported. Focusing on specific subgroups, patients with active AIDs seemed to have higher ORR (50\%) compared with patients with inactive AIDs (38.1\%) and those without (35.3\%) AIDs. Conversely, a not significant trend toward a worse median PFS has been observed in patients with active (6.8 months) $v s$. inactive (14.4 months) AIDs (58). Similarly, worse median OS has been reported in patients with active (9.8 months) compared to inactive (15.7 months) or no (16.5 months) AIDs, likely due to the impact of the disease on life expectancy, being aware that the transient interruptions of immunotherapy due to toxicity should not affect OS $(26,59)$.

Interestingly, patients with ECOG-PS $>2$ seemed to have a lower incidence of irAEs, likely due to their poor general conditions, which may have impaired the reactivity of their immune system (59).

Another study published in 2018 in $7 C O$, analyzed a total of 56 patients diagnosed with NSCLC and AIDs undergoing immunotherapy. About $55 \%$ of patients developed irAEs (38\%) and/or AIDs' exacerbation (23\%). The irAEs were mostly of mild grade (74\%) but in $26 \%$ of cases, a severe irAE has been reported. Half of them did not require immunosuppressive therapy for symptom control and, the majority of the remaining patients were treated with only corticosteroids. As regards AIDs' exacerbations, they were G1-G2 in $87 \%$ of cases, and G3 in the remaining $13 \%$ of cases, while no G4 events occurred. Importantly exacerbations were more frequent in patients with symptomatic versus non-symptomatic AIDs (50\% vs. 18\%) at the time of immunotherapy initiation. Furthermore, no difference in flares incidence was found between patients assuming an immunosuppressive drug at baseline and those who did not, while in the $5 \%$ of cases a concomitance of AIDs and irAEs flares has been described.

In this study, the overall percentage of G3-G4 irAEs was found to be $11 \%$, in line with that (7-15\%) emerging from the CPIs registration studies, which did not include 
AIDs affected patients. However, the rate of treatment discontinuation due to immune related toxicity was significantly higher (14\% vs. 3-8\%) (60).

A poster presented at ASCO 2019 by Sean Khozin showed the results of a retrospective observational cohort study including 2,425 patients with stage III-IV NSCLC, who received at least one dose of CPI therapy. A diagnosis of AID was reported in $22 \%$ of patients $(n=538)$, whose median survival was similar to that observed in the remaining population not affected by AIDs. Unlike the previous evidence, the results of this study did not show increased risk of developing irAEs in the AIDs-affected subgroup (64).

Similarly, a recent retrospective study including 29 CPIs' treated patients with advanced lung cancer (adenocarcinoma $59 \%$, squamous cell carcinoma $34 \%$, small cell cancer $7 \%$, undifferentiated $3 \%$ ), showed a not-increased incidence of irAEs in patients with AIDs compared to the control population. Furthermore, no significant survival differences have been observed according to both irAE occurrence and baseline immunomodulatory therapy (65). However, another retrospective study including 112 cancer patients with AIDs treated with immunotherapy (anti-PD-1/PDL1 83\%, anti-CTLA-4 13\%, combination 3\%), showed a reduced PFS in patients who developed immunotoxicity (irAEs or AID flares), as compared to the control cohort (HR 1.97, $\mathrm{P}=0.032)(61)$.

An international multicenter retrospective study analyzed 102 cancer patients with a concomitant diagnosis of inflammatory bowel disease (IBD) who were treated with immunotherapeutic drugs. Among these, 49 patients suffered from Crohn's disease, 49 from ulcerative colitis and 4 from unclassified inflammatory disease, while the control group consisted of 11,377 cancer patients with no IBD receiving immunotherapy. The most represented tumor was melanoma (44\%) followed by NSCLC (23\%) and gastrointestinal (GI) tumors (17\%), with $83 \%$ of patients receiving an anti $\mathrm{PD}-1 / \mathrm{PD}-\mathrm{L} 1$ agent. Among the 102 analyzed patients, $42(41 \%)$ presented a GI side effect, with a median incidence of 62 days from the treatment initiation, and 23 of them discontinued immunotherapy. Forty-one patients developed diarrhea, of grade 3-4 in about half of cases, and $29 \%$ required the administration of nonsteroidal immunosuppressive drugs, such as Infliximab and Vedolizumab. Due to the side effects, 32 patients underwent endoscopic investigation leading to the detection of mucosal ulceration in $38 \%$ and of non-ulcerative inflammation in $44 \%$ of cases. A total of 4 patients underwent a colic perforation. In the control group, the incidence of GI side effects was significantly lower (11\%). Of the 42 patients who developed toxicities, 15 presented a second episode during the follow-up period. Patients who had active IBD in the three months prior to immunotherapy initiation experienced higher grade diarrhea than patients with inactive IBD. No difference in severity of GI adverse effects was observed between patients who were being treated for IBD at the beginning of immunotherapy and those who were not. The tumor response to CPIs' therapy was similar between patients affected by IBD and those who did not, suggesting the effectiveness of immunotherapy treatment even in this particular population (62).

\section{Critical discussion}

The major concerns about the use of immunotherapy in NSCLC patients with pre-existing AIDs was related to an increased risk of developing serious irAEs and a flare-up of their baseline pathological conditions, as compared to the general cancer population (4-6).

The occurrence of irAEs has been reported to be higher in patients with pre-existing AIDs compared to the control population, however the majority of irAEs are mild and well-controlled by corticosteroids use, with a limited number of patients requiring non-steroidal immunosuppressive drugs for high grade toxicities $(60,61)$. A transient discontinuation of CPIs' therapy $(59,61,65)$ due to irAEs occurrence may be required, but, according to available evidence, no significant increased risk of highgrade irAEs leading to permanent suspension of CPIs' treatment has been reported. Particularly the percentage of NSCLC patients experiencing high-grade irAEs and CPIs' treatment discontinuation were $11 \%$ and $14 \%$, respectively, thus very similar to those reported in clinical trials not including AIDs population. Conversely the risk of developing AID flare seems to be related to the presence of AID symptoms at the time of CPIs initiation with a mild prevalence among patients diagnosed with rheumatologic AID (60). Therefore, particular attention should be paid to the clinical management of patients with active and symptomatic preexisting AIDs who are candidate to receiving CPIs' therapy. As regards the immunotherapy effectiveness in this special population, the available evidence suggested a similar activity of CPIs' therapy in NSCLC patients with or without AIDs, with worse OS limited to symptomatic AIDs cases.

In the majority of the aforementioned studies (Table 2) 
CPIs have been administered in pre-treated patients, thus leading to a potential underestimation of the real incidence of irAEs in this special population considering that the long lasting and widespread use of immunotherapy in first-line may be associated to higher risk of developing irAEs, especially in those patients with pre-existing AIDs. Similarly, the survival outcomes reported in these studies (Table 2) should be carefully interpreted as they reflect the health status of cancer patients who had previously received other cancer treatments, whose influence on immune reactivity is not known and established yet.

Comforting data about the use of immunotherapy also comes from studies conducted on patients with IBD, to whom the treatment benefits seem to outweigh the risks. However, patients with active IBD at the time of immunotherapy initiation, seem to have higher risk of developing severe immune mediated GI adverse events and, therefore require to be early identified and adequately managed and treated, if necessary. In this regard, the early introduction of immunosuppressive drugs such as Infliximab and Vedolizumab has been indeed associated to better survival outcomes, especially in those patients with a severe endoscopic presentation (62).

According to Haanen et al., patients with autoimmune disorders and cancer requiring immunotherapy could benefit from a 2-step approach with personalized therapy depending on the autoimmune pathology itself. The suggested algorithm foresees a first phase lasting 1 month, whose objective is to control the autoimmune pathology with specific immunosuppressive drugs, limiting the use and dose of corticosteroids, and a second phase in which the immunotherapeutic drug may be introduced. The periodic follow-up aims to evaluate any chemical alterations in the laboratory and clinic to detect any exacerbations at an early stage and intervene with specific targeted therapies (66).

Finally, a 2019 Japanese study investigated the possible influence of antinuclear antibody (ANA) positivity on the incidence of irAEs. The authors demonstrated that $\mathrm{ANA}^{+}$patients did not present a higher risk of developing irAEs compared with negative patients but showed shorter PFS and OS. This is likely due to the underlying chronic inflammatory status, which is a known factor influencing carcinogenesis $(67,68)$. Furthermore, 10 out of 18 baseline $\mathrm{ANA}^{+}$patients recruited in the study were monitored for ANA titer during CPIs' treatment, with 3 of them showing an increase of this value and a subsequent development of irAEs. Although very preliminary, these data suggested that a dynamic monitoring of ANA value could play a potential role in the measurement of patient immunoreactivity, as predictive factor for the development of irAEs (67).

Ongoing prospective studies are currently investigating the efficacy and safety profile of immunotherapy in NSCLC diagnosed with AIDs (NCT03656627, NCT03816345).

\section{Conclusions}

The real impact of both concomitant steroids therapy and AIDs on immunotherapy efficacy/tolerability in NSCLC patients remains an actual and controversial topic requiring prospective investigation, in order to develop standardized selection criteria and shared recommendations for their clinical management. In the current scenario, characterized by limited knowledge and lack of dedicated guidelines, an international panel of experts from the International Association for the Study of Lung Cancer (IASLC) have recently provided practical suggestions which may guide physicians in their current practice (69). Therefore, according to the IASLC panel: "not all patients with metastatic NSCLC receiving concomitant steroids treatment should be excluded from CPIs therapy and discontinuation of steroids is not always required at the time of CPIs initiation". Similarly, "CPIs' therapy may be offered to NSCLC patients affected by nonlife-threatening and asymptomatic AIDs". Finally, a clinical decision on each individual patient is currently suggested and a close clinical monitoring highly recommended.

\section{Acknowledgments}

Funding: None.

\section{Footnote}

Provenance and Peer Review: This article was commissioned by the Guest Editors (Jordi Remon and Benjamin Besse) for the series "Immunotherapy in other thoracic malignancies and uncommon populations" published in Translational Lung Cancer Research. The article has undergone external peer review.

Conflicts of Interest: All authors have completed the ICMJE uniform disclosure form (available at http://dx.doi. org/10.21037/tlcr-20-635). The series "Immunotherapy in other thoracic malignancies and uncommon populations" was commissioned by the editorial office without any funding or sponsorship. FP reports personal fees from MSD, personal fees from Boehringer Ingelheim, personal 
fees from Astra Zeneca, outside the submitted work. SN reports personal fees from Eli Lilly, personal fees from MSD, personal fees from Roche, personal fees from BMS, personal fees from Takeda, personal fees from Pfizer, personal fees from Astra Zeneca, personal fees from Boehringer Ingelheim, outside the submitted work. The authors have no other conflicts of interest to declare.

Ethical Statement: The authors are accountable for all aspects of the work in ensuring that questions related to the accuracy or integrity of any part of the work are appropriately investigated and resolved.

Open Access Statement: This is an Open Access article distributed in accordance with the Creative Commons Attribution-NonCommercial-NoDerivs 4.0 International License (CC BY-NC-ND 4.0), which permits the noncommercial replication and distribution of the article with the strict proviso that no changes or edits are made and the original work is properly cited (including links to both the formal publication through the relevant DOI and the license). See: https://creativecommons.org/licenses/by-nc-nd/4.0/.

\section{References}

1. Siegel RL, Miller KD, Jemal A. Cancer statistics, 2018. CA Cancer J Clin 2018;68:7-30.

2. Brahmer J, Reckamp KL, Baas P, et al. Nivolumab versus docetaxel in advanced squamous-cell non-small-cell lung cancer. N Engl J Med 2015;373:123-35.

3. Borghaei H, Paz-Ares L, Horn L, et al. Nivolumab versus docetaxel in advanced nonsquamous non-small-cell lung cancer. N Engl J Med 2015;373:1627-39.

4. Herbst RS, Baas P, Kim DW, et al. Pembrolizumab versus docetaxel for previously treated, $\mathrm{PD}-\mathrm{L} 1$-positive, advanced non-small-cell lung cancer (KEYNOTE-010): A randomised controlled trial. Lancet 2016;387:1540-50.

5. Rittmeyer A, Barlesi F, Waterkamp D, et al. Atezolizumab versus docetaxel in patients with previously treated non-small-cell lung cancer (OAK): a phase 3, openlabel, multicentre randomised controlled trial. Lancet 2017;389:255-65.

6. Reck M, Rodríguez-Abreu D, Robinson AG, et al. Pembrolizumab Versus Chemotherapy for PD-L1Positive Non-Small-Cell Lung Cancer. N Engl J Med 2016;375:1823-33.

7. Brahmer JR, Rodríguez-Abreu D, Robinson AG, et al. Health-related quality-of-life results for pembrolizumab versus chemotherapy in advanced, PD-L1-positive NSCLC (KEYNOTE-024): a multicentre, international, randomised, open-label phase 3 trial. Lancet Oncol 2017;18:1600-9.

8. Garon EB, Hellmann MD, Rizvi NA, et al. Five-Year Overall Survival for Patients With Advanced Non SmallCell Lung Cancer Treated With Pembrolizumab: Results From the Phase I KEYNOTE-001Study. J Clin Oncol 2019;37:2518-27.

9. Paz-Ares L, Luft A, Vicente D, et al. Pembrolizumab plus chemotherapy for squamous non-small-cell lung cancer. $\mathrm{N}$ Engl J Med 2018;379:2040-51.

10. Gandhi L, Rodríguez-Abreu D, Gadgeel S, et al. Pembrolizumab plus chemotherapy in metastatic nonsmall-cell lung cancer. N Engl J Med 2018;378:2078-92.

11. Socinski MA, Jotte RM, Cappuzzo F, et al. Atezolizumab for first-line treatment of metastatic nonsquamous NSCLC. N Engl J Med 2018;378:2288-301.

12. Wang Y, Zhou S, Yang F, et al. Treatment-Related Adverse Events of PD-1 and PD-L1 Inhibitors in Clinical Trials: A Systematic Review and Meta-analysis. JAMA Oncol 2019;5:1008-19.

13. Nosaki K, Saka H, Hosomi Y, et al. Safety and efficacy of pembrolizumab monotherapy in elderly patients with PDL1-positive advanced non-small-cell lung cancer: Pooled analysis from the KEYNOTE-010, KEYNOTE-024, and KEYNOTE-042 studies. Lung Cancer 2019;135:188-95.

14. Facchinetti F, Mazzaschi G, Barbieri F, et al. First-line pembrolizumab in advanced non-small cell lung cancer patients with poor performance status. Eur J Cancer 2020;130:155-67.

15. Mansfield AS, Herbst RS, Castro G, et al. Outcomes with pembrolizumab (pembro) monotherapy in patients (pts) with PD-L1-positive NSCLC with brain metastases: Pooled analysis of KEYNOTE-001, -010, -024, and -042. Ann Oncol 2019;30:v604-6.

16. Ryken TC, McDermott M, Robinson PD, et al. The role of steroids in the management of brain metastases: A systematic review and evidence-based clinical practice guideline. J Neurooncol 2010;96:103-14.

17. Paulsen Ø, Klepstad P, Rosland JH, et al. Efficacy of methylprednisolone on pain, fatigue, and appetite loss in patients with advanced cancer using opioids: A randomized, placebo-controlled, double-blind trial. J Clin Oncol 2014;32:3221-8.

18. Yennurajalingam S, Frisbee-Hume S, Palmer JL, et al. Reduction of cancer-related fatigue with dexamethasone: A double-blind, randomized, placebo-controlled 
trial in patients with advanced cancer. J Clin Oncol 2013;31:3076-82.

19. Ben-Aharon I, Gafter-Gvili A, Leibovici L, et al. Interventions for alleviating cancer-related dyspnea: A systematic review and meta-analysis. Acta Oncol 2012;51:996-1008.

20. Lin RJ, Adelman RD, Mehta SS. Dyspnea in palliative care: Expanding the role of corticosteroids. J Palliat Med 2012;15:834-7.

21. Franchimont D. Overview of the actions of glucocorticoids on the immune response: A good model to characterize new pathways of immunosuppression for new treatment strategies. Ann N Y Acad Sci 2004;1024:124-37.

22. Flint TR, Janowitz T, Connell CM, et al. Tumor-Induced IL-6 Reprograms Host Metabolism to Suppress Antitumor Immunity. Cell Metab 2016;24:672-84.

23. Connell CM, Raby S, Beh I, et al. Cancer immunotherapy trial registrations increase exponentially but chronic immunosuppressive glucocorticoid therapy may compromise outcomes. Ann Oncol 2017;28:1678-9.

24. Hinrichs CS, Palmer DC, Rosenberg SA, et al. Glucocorticoids do not inhibit antitumor activity of activated CD8+ T cells. J Immunother 2005;28:517-24.

25. Franchimont D, Kino T, Galon J, et al. Glucocorticoids and inflammation revisited: the state of the art. NIH clinical staff conference. Neuroimmunomodulation 20022003;10:247-60.

26. Schadendorf D, Wolchok JD, Hodi FS, et al. Efficacy and Safety Outcomes in Patients With Advanced Melanoma Who Discontinued Treatment With Nivolumab and Ipilimumab Because of Adverse Events: A Pooled Analysis of Randomized Phase II and III Trials. J Clin Oncol 2017;35:3807-14.

27. Postow MA, Sidlow R, Hellmann MD. Immune-Related Adverse Events Associated with Immune Checkpoint Blockade. N Engl J Med 2018;378:158-68.

28. Horvat TZ, Adel NG, Dang TO, et al. Immune-related adverse events, need for systemic immunosuppression, and effects on survival and time to treatment failure in patients with melanoma treated with ipilimumab at memorial sloan kettering cancer center. J Clin Oncol 2015;33:3193-8.

29. Leighl N, Gandhi L, Hellmann MD, et al. Pembrolizumab for NSCLC: Immune-mediated adverse events and corticosteroid use. J Thorac Oncol 2015;10:S233.

30. Von Pawel J, Syrigos K, Mazieres J, et al. Association between immune-related adverse events (irAEs) and atezolizumab efficacy in advanced NSCLC: analyses from the phase III study OAK. Ann Oncol 2017;28:v469.
31. Santini FC, Rizvi H, Plodkowski AJ, et al. Safety and Efficacy of Re-treating with Immunotherapy after Immune-Related Adverse Events in Patients with NSCLC. Cancer Immunol Res 2018;6:1093-9.

32. Arbour KC, Mezquita L, Long N, et al. Impact of baseline steroids on efficacy of programmed cell death-1 and programmed death-ligand 1 blockade in patients with nonsmall-cell lung cancer. J Clin Oncol 2018;36:2872-8.

33. Scott SC, Pennell NA. Early Use of Systemic Corticosteroids in Patients with Advanced NSCLC Treated with Nivolumab. J Thorac Oncol 2018;13:1771-5.

34. Fucà G, Galli G, Poggi M, et al. Modulation of peripheral blood immune cells by early use of steroids and its association with clinical outcomes in patients with metastatic non-small cell lung cancer treated with immune checkpoint inhibitors. ESMO Open 2019;4:1-8.

35. Pan EY, Merl MY, Lin K. The impact of corticosteroid use during anti-PD1 treatment. J Oncol Pharm Pract 2020;26:814-22.

36. Martinez JM, Riuvadets M, García-Campelo MR, et al. Impact of corticosteroids and antibiotics on efficacy of immune-checkpoint inhibitors in advanced non-small cell lung cancer. Ann Oncol 2019;30:v525.

37. Drakaki A, Luhn P, Wakelee H, et al. Association of systemic corticosteroids with overall survival in patients receiving cancer immunotherapy for advanced melanoma, non-small cell lung cancer or urothelial cancer in routine clinical practice. Ann Oncol 2019;30:xi16-xi17.

38. Patil P, Jia X, Hobbs B, et al. MA03.01 The Impact of Early Steroids on Clinical Outcomes in Patients with Advanced NSCLC Treated with Immune Checkpoint Inhibitors- A Cancerlinq Cohort. J Thorac Oncol 2019;14:S256.

39. Wakuda K, Miyawaki T, Miyawaki E, et al. The impact of steroid use on efficacy of immunotherapy among patients with lung cancer who have developed immune-related adverse events. J Clin Oncol 2019;37:e20583.

40. Ricciuti B, Dahlberg SE, Adeni A, et al. Immune Checkpoint Inhibitor Outcomes for Patients With Non-Small-Cell Lung Cancer Receiving Baseline Corticosteroids for Palliative Versus Nonpalliative Indications. J Clin Oncol 2019;37:1927-34.

41. De Giglio A, Mezquita L, Auclin E, et al. Impact of early introduction of steroid on immune-checkpoint inhibitors (ICI) in patients with advanced non-small cell lung cancer treated. Ann Oncol 2019;30:xi16.

42. Flammer JR, Rogatsky I. Minireview: Glucocorticoids in autoimmunity: Unexpected targets and mechanisms. Mol 
Endocrinol 2011;25:1075-86.

43. Taube JM, Anders RA, Young GD, et al. Colocalization of inflammatory response with B7-h1 expression in human melanocytic lesions supports an adaptive resistance mechanism of immune escape. Sci Transl Med 2012;4:127ra37.

44. Tumeh PC, Harview CL, Yearley JH, et al. PD-1 blockade induces responses by inhibiting adaptive immune resistance. Nature 2014;515:568-71.

45. Kansy BA, Concha-Benavente F, Srivastava RM, et al. PD-1 Status in CD8+ T Cells Associates with Survival and Anti-PD-1 Therapeutic Outcomes in Head and Neck Cancer. Cancer Res 2017;77:6353-64.

46. Wei SC, Levine JH, Cogdill AP, et al. Distinct Cellular Mechanisms Underlie Anti-CTLA-4 and Anti-PD-1 Checkpoint Blockade. Cell 2017;170:1120-1133.e17.

47. Cattermole GN, Leung PYM, Ho GYL, et al. The normal ranges of cardiovascular parameters measured using the ultrasonic cardiac output monitor. Physiol Rep 2017;5:e13195.

48. Maxwell R, Luksik AS, Garzon-Muvdi T, et al. Contrasting impact of corticosteroids on anti-PD-1 immunotherapy efficacy for tumor histologies located within or outside the central nervous system. Oncoimmunology 2018;7:e1500108.

49. Giles AJ, Hutchinson MND, Sonnemann HM, et al. Dexamethasone-induced immunosuppression: mechanisms and implications for immunotherapy. J Immunother Cancer 2018;6:51.

50. Phan GQ, Yang JC, Sherry RM, et al. Cancer regression and autoimmunity induced by cytotoxic $\mathrm{T}$ lymphocyteassociated antigen 4 blockade in patients with metastatic melanoma. Proc Natl Acad Sci U S A 2003;100:8372-7.

51. Harmankaya K, Erasim C, Koelblinger C, et al. Continuous systemic corticosteroids do not affect the ongoing regression of metastatic melanoma for more than two years following ipilimumab therapy. Med Oncol 2011;28:1140-4.

52. Beck KE, Blansfield JA, Tran KQ, et al. Enterocolitis in patients with cancer after antibody blockade of cytotoxic T-lymphocyte-associated antigen 4. J Clin Oncol 2006;24:2283-9.

53. Attia P, Phan GQ, Maker A V, et al. Autoimmunity correlates with tumor regression in patients with metastatic melanoma treated with anti-cytotoxic T-lymphocyte antigen-4. J Clin Oncol 2005;23:6043-53.

54. Downey SG, Klapper JA, Smith FO, et al. Prognostic factors related to clinical response in patients with metastatic melanoma treated by CTL-associated antigen-4 blockade. Clin Cancer Res 2007;13:6681-8.

55. Margolin K, Ernstoff MS, Hamid O, et al. Ipilimumab in patients with melanoma and brain metastases: an openlabel, phase 2 trial. Lancet Oncol 2012;13:459-65.

56. Khan SA, Pruitt SL, Xuan L, et al. Prevalence of Autoimmune Disease Among Patients With Lung Cancer: Implications for Immunotherapy Treatment Options. JAMA Oncol 2016;2:1507-8.

57. Simon TA, Thompson A, Gandhi KK, et al. Incidence of malignancy in adult patients with rheumatoid arthritis: a meta-analysis. Arthritis Res Ther 2015;17:212. Erratum in: Arthritis Res Ther. 2016 May 4;18(1):100. doi: 10.1186/ s13075-016-0990-5.

58. Danlos FX, Voisin AL, Dyevre V, et al. Safety and efficacy of anti-programmed death 1 antibodies in patients with cancer and pre-existing autoimmune or inflammatory disease. Eur J Cancer 2018;91:21-9.

59. Cortellini A, Buti S, Santini D, et al. Clinical Outcomes of Patients with Advanced Cancer and Pre-Existing Autoimmune Diseases Treated with Anti-Programmed Death-1 Immunotherapy: A Real-World Transverse Study. Oncologist 2019;24:e327-e337.

60. Leonardi GC, Gainor JF, Altan M, et al. Safety of Programmed Death-1 Pathway Inhibitors Among Patients With Non-Small-Cell Lung Cancer and Preexisting Autoimmune Disorders. J Clin Oncol 2018;36:1905-12.

61. Tison A, Quéré G, Misery L, et al. Safety and Efficacy of Immune Checkpoint Inhibitors in Patients With Cancer and Preexisting Autoimmune Disease: A Nationwide, Multicenter Cohort Study. Arthritis Rheumatol 2019;71:2100-11.

62. Abu-Sbeih H, Faleck DM, Ricciuti B, et al. Immune checkpoint inhibitor therapy in patients with preexisting inflammatory bowel disease. J Clin Oncol 2020;38:576-83.

63. Khan SA, Pruitt SL, Xuan L, et al. How does autoimmune disease impact treatment and outcomes among patients with lung cancer? A national SEER-Medicare analysis. Lung Cancer 2018;115:97-102.

64. Khozin S, Zhi J, Jun M t al. Real-world characteristics and outcomes of patients with advanced non-small cell lung cancer (aNSCLC) receiving immune checkpoint inhibitor. J Clin Oncol 2019;37:9110.

65. Cytryn S, Efuni E, Velcheti V, et al. P2.04-48 Use of Immune Checkpoint Inhibitors in Patients with Advanced Lung Cancer and Pre-Existing Autoimmune Diseases. J Thorac Oncol 2019;14:S726-7.

66. Haanen J, Ernstoff MS, Wang Y, et al. Autoimmune 
diseases and immune-checkpoint inhibitors for cancer therapy: review of the literature and personalized riskbased prevention strategy. Ann Oncol 2020;31:724-44.

67. Yoneshima Y, Tanaka K, Shiraishi Y, et al. Safety and efficacy of PD-1 inhibitors in non-small cell lung cancer patients positive for antinuclear antibodies. Lung Cancer 2019;130:5-9.

Cite this article as: Passiglia F, Cetoretta V, De Filippis M, Napoli V, Novello S. Exploring the immune-checkpoint inhibitors' efficacy/tolerability in special non-small cell lung cancer (NSCLC) populations: focus on steroids and autoimmune disease. Transl Lung Cancer Res 2021;10(6):28762889. doi: $10.21037 /$ tlcr-20-635
68. Murata M. Inflammation and cancer. Environ Health Prev Med 2018;23:50.

69. Remon J, Passiglia F, Ahn MJ, et al. Immune Checkpoint Inhibitors in Thoracic Malignancies: Review of the Existing Evidence by an IASLC Expert Panel and Recommendations. J Thorac Oncol 2020;15:914-47. 